American Journal of Applied Sciences 9 (5): 620-623, 2012

ISSN 1546-9239

(C) 2012 Science Publications

\title{
Visual Contact between Two Earth's Satellites
}

\author{
${ }^{1}$ Awad, M.E., ${ }^{2}$ M.A. Sharaf and ${ }^{1}$ E.H. Khatab \\ ${ }^{1}$ Departmant of Astronomy, Space and Meteorology, \\ Faculty of Science, Cairo University, 12613, Egypt \\ ${ }^{2}$ Departmant of Astronomy, Faculty of Science, \\ King Abd El-Aziz University, Jeddah, Saudi Arabia
}

\begin{abstract}
Problem statement: In this study, an analytical expression for predicting mutually visible between Earth's satellites is developed. Approach: A relative satellite-satellite visibility function is developed in terms of orbital elements for two satellites in the same orbital plane. Also, a simple computational algorithm is expressed to compute the visibility function. Results: This study presents a new and easy method to determine the visibility times between two satellites which are in the same orbital plane. Conclusion: The satellite-satellite visibility function is solved by an easy treatment.
\end{abstract}

Key words: Satellite-satellite visibility, satellite-ground station visibility, rise-set function

\section{INTRODUCTION}

Mutually visible satellites are defined as two artificial satellites that can maintain direct line of sight between each other for a certain time. Actually, the analysis to be developed is primarily concerned with the rise and set time of a given satellite with respect to another, that is, the time of loss or gain of direct line of sight.

The desirability of having a simple analytic method for predicting when two or more satellites are mutually visible to each other is important for examples in (satellite communications, space warfare).

Escobal proposed a faster method to solve the satellite versus Earth-fixed tracking station problem by developing a closed-form solution for the visibility periods about an oblate Earth. He transformed the satellite and tracking station geometry into a single transcendental equation as a function of eccentric anomaly (Escobal, 1964). Roth developed a method to determine the rise-set time and maximum elevation of a satellite (Roth, 1976). Lawton developed a method to solve for satellite-satellite and satellite-ground station visibility periods for vehicles in circular or near circular orbits by approximating the visibility function with a Fourier series. He determined the local periodicity of this curve and then used a numerical search to locate rise-set times (Lawton, 1986). Alfano presented a numerical method to rapidly determine the rise-set times of satellite-satellite and satellite-ground station visibility periods, with the line-of-sight corrected for an oblate Earth. They calculated the problem by using an algorithm which used a space curve modeling technique known as parabolic blending to construct the waveform of the visibility function versus time. The algorithm was worked for all orbital eccentricities and perturbed satellite motion and was provided the visibility function to not become discontinuous as it could from thrusting or deploying a tether (Alfano et al., 1992).

In this study, we develop an analytical expression for predicting the visibility function when two satellites are mutually visible to each other. Also, this visibility function will reduce to a function in terms of the orbital elements when the two satellites are in the same plane., we express a computational algorithm to compute this visibility function.

\section{MATERIALS AND METHODS}

Development of visibility function:

Relative visibility geometry: Consider the geometry defined in Fig. 1. In which Sat. 1 and Sat. 2 are visible to each other. Where $\vec{r}_{1}$ and $\vec{r}_{2}$ are the position vectors of the Sat. 1 and Sat. 2 respectively; $\overline{\mathrm{S}}$ emanates from the Earth's dynamical center perpendicular to $\overrightarrow{\mathrm{C}}=\left(\overrightarrow{\mathrm{x}}_{1}+\overrightarrow{\mathrm{x}}_{2}\right)$ at $\mathrm{h}$ and intersect the Earth's surface at $\mathrm{q}$, where $\overrightarrow{\mathrm{x}}_{1}$ and $\overrightarrow{\mathrm{x}}_{2}$ are unknown vectors, $\overrightarrow{\mathrm{x}}_{1}$ is the distance vector from Sat. 1 to $h$ and $\vec{x}_{2}$ is the distance vector from h to Sat. 2. The magnitude of $\overline{\mathrm{S}}$ is divided into $|\overrightarrow{\mathrm{oq}}|=\mathrm{R}_{\mathrm{E}}$ which is the Earth's radius and $|\overrightarrow{\mathrm{qh}}|=\mathrm{R}_{\mathrm{V}}$ which is the thickness of the atmosphere from the Earth's surface to the vector $\overrightarrow{\mathrm{C}}$, it follows as:

$$
S^{2}=\vec{S} . \vec{S}=\left(R_{E}+R_{V}\right)^{2}
$$




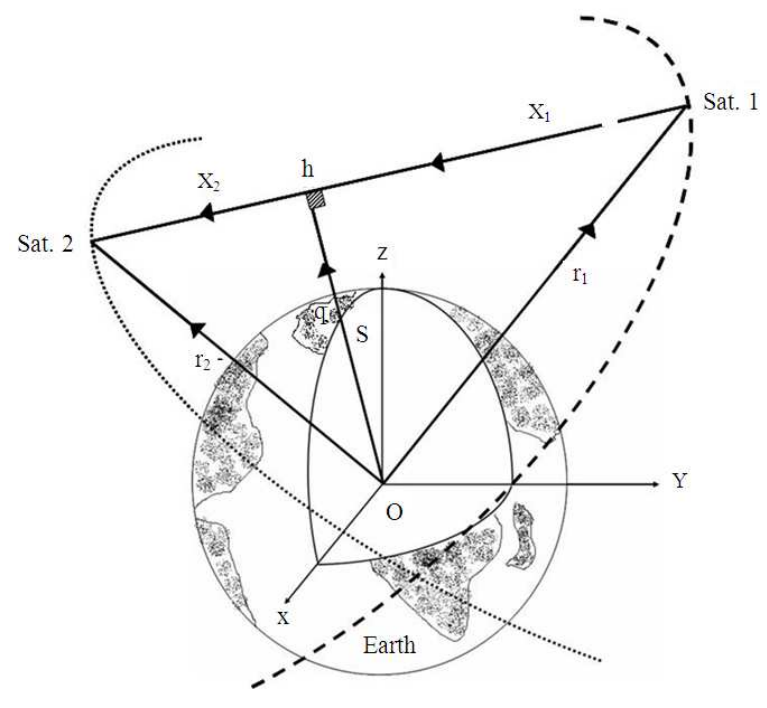

Fig. 1: The geometry of two satellites in case of direct line-of-sight

Analytical expression of the relative visibility function: Examination of Fig. 1 allows the two fundamental vector closure equations to be written as:

$\overrightarrow{\mathrm{r}}_{1}=\overrightarrow{\mathrm{S}}-\overrightarrow{\mathrm{x}}_{1} \quad, \quad \overrightarrow{\mathrm{r}}_{2}=\overrightarrow{\mathrm{S}}+\overrightarrow{\mathrm{x}}_{2}$

The magnitudes of $\vec{x}_{1}$ and $\vec{x}_{2}$ can be determined at once by dotting Eq. 1 on themselves as

$\overrightarrow{\mathrm{r}}_{1}^{2}=\mathrm{S}^{2}+\mathrm{x}_{1}^{2}+2\left(\overrightarrow{\mathrm{S}} \cdot \overrightarrow{\mathrm{x}}_{1}\right), \mathrm{r}_{2}^{2}=\mathrm{S}^{2}+\mathrm{x}_{2}^{2}+2\left(\overrightarrow{\mathrm{S}}_{\mathrm{x}}\right)$

Hnece $\overrightarrow{\mathrm{S}}$ is perpendicular on $\overrightarrow{\mathrm{C}}$ then we have:

$\overrightarrow{\mathrm{S}} \cdot \overrightarrow{\mathrm{x}}_{1}=\overrightarrow{\mathrm{S}} \cdot \overrightarrow{\mathrm{x}}_{2}=0$

thus Eq. 2 can be written as:

$\mathrm{x}_{1}=\sqrt{\mathrm{r}_{1}^{2}-\mathrm{S}^{2}}, \quad \mathrm{x}_{2}=\sqrt{\mathrm{r}_{2}^{2}-\mathrm{S}^{2}}$

From Fig. 1 we have:

$\overrightarrow{\mathrm{C}}=\overrightarrow{\mathrm{r}}_{2}-\overrightarrow{\mathrm{r}}_{1}$

Then:

$\mathrm{C}=\sqrt{\mathrm{r}_{1}^{2}+\mathrm{r}_{2}^{2}-2 \alpha}$

Where:

$\alpha=r_{1} \cdot r_{2}$

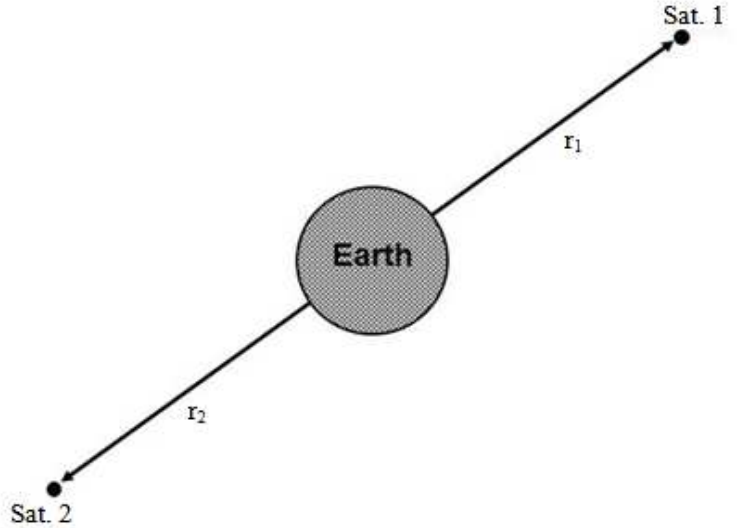

Fig. 2: The geometry of the case in which direct line-ofsight visibility is impossibility

Also, from Eq. 3 we have:

$\mathrm{C}=\mathrm{x}_{1}+\mathrm{x}_{2}=\sqrt{\mathrm{r}_{1}^{2}-\mathrm{S}^{2}}+\sqrt{\mathrm{r}_{2}^{2}-\mathrm{S}^{2}}$

Equating Eq. 4 and 6 and then squaring twice, we get:

$\therefore \alpha^{2}-\mathrm{r}_{1}^{2} \mathrm{r}_{2}^{2}+\mathrm{S}^{2}\left(\mathrm{r}_{1}^{2}+\mathrm{r}_{2}^{2}\right)-2 \mathrm{~S}^{2} \alpha=0$

It is then possible to obtain an analytical expression for the visibility function, with using Eq. 5, as:

$$
\mathrm{R}_{\mathrm{V}}=\left(\sqrt{\frac{\mathrm{r}_{1}^{2} \mathrm{r}_{2}^{2}-\left(\overrightarrow{\mathrm{r}}_{1}, \overrightarrow{\mathrm{r}}_{2}\right)^{2}}{\mathrm{r}_{1}^{2}+\mathrm{r}_{2}^{2}-2\left(\overrightarrow{\mathrm{r}}_{1}, \overrightarrow{\mathrm{r}}_{2}\right)}}\right)-\mathrm{R}_{\mathrm{E}}
$$

Nonvisibility using the visibility function $\left(\mathbf{R}_{\mathrm{V}}\right)$ : The visibility function defined by Eq. 8 can be used to predict explicitly whether or not satellites are visible to one another. The sign of $R_{V}$ associated with visibility can be obtained by constructing a case in which direct line-of-sight visibility is impossibility as shown in Fig. 2. For this case we have:

$\overline{\mathrm{r}_{1}} \cdot \overline{\mathrm{r}}_{2}=\mathrm{r}_{1} \mathrm{r}_{2} \cos (180)=-\mathrm{r}_{1} \mathrm{r}_{2}$

Then Eq. 8 can be written as to:

$\mathrm{R}_{\mathrm{v}}=-\mathrm{R}_{\mathrm{E}}$

Hence, we can put this rule:

$\mathrm{R}_{\mathrm{v}}=\left\{\begin{array}{ccc}\text { Positive value } & \rightarrow \text { Derect line }- \text { of }- \text { sight } \\ \text { zero } & \rightarrow & \text { rise or set } \\ \text { Negative value } & \rightarrow & \text { Nonvisibility Case }\end{array}\right.$ 
Reduction of the visibility function using the orbital elements: In terms of the orbital eccentricity e, semiparameter $p$ and true anomaly $f$, the equation of each orbit can be expressed by the relationship:

$$
r_{i}=\frac{p_{i}}{\left(1+e_{i} \cos f_{i}\right)^{\prime}} \quad i=1,2
$$

When the two satellites are in the same orbital plane: In this case, Fig. 3 shows when the two satellites are in the same orbital plane, then the two satellites has the same inclination and longitude of ascending node.

By using Eq. 9:

$$
\vec{r}_{1} \cdot \vec{r}_{2}=\frac{p_{1} p_{2} \cos \left(f_{2}-f_{1}\right)}{\left(1+e_{1} \cos f_{1}\right)\left(1+e_{2} \cos f_{2}\right)}
$$

Substituting from Eq. 9 and 10 into Eq. 8:

$$
R_{v}=\left(\sqrt{\frac{p_{1} p_{2} \sin ^{2}\left(f_{2}-f_{1}\right)}{\varepsilon}}\right)-R_{E}
$$

Where:

$$
\begin{aligned}
\varepsilon & =-2 p_{1} p_{2} \cos \left(f_{2}-f_{1}\right)\left(1+e_{1} \cos f_{1}\right)\left(1+e_{2} \cos f_{2}\right) \\
& +p_{2}^{2}\left(1+e_{1} \cos f_{1}\right)^{2}+p_{1}^{2}\left(1+e_{2} \cos f_{2}\right)^{2}
\end{aligned}
$$

\section{Computational algorithm:}

Purpose: Mutual visibility between two Earth's satellites in the same orbital planes.

Input: Two line elements of the two satellites, $t$ (time), $\mathrm{i}=1,2$

\section{Computational sequence:}

- Calculate $\mathrm{M}_{\mathrm{i}}=\mathrm{n}_{\mathrm{i}}\left(\mathrm{t}-\mathrm{T}_{\mathrm{i}}\right)$

- Solve for $\mathrm{E}_{\mathrm{i}}$ from Kepler's equation.

- Calculate $f_{i}=\tan ^{-1}\left(\frac{-\left(\sin E_{i} \sqrt{\left(1-e_{i}^{2}\right)}\right)}{\left(e_{i}-\cos E_{i}\right)}\right)$

- Calculate $r_{i}=a_{i}\left(1-e_{i} \cos E_{i}\right)$

- Calculate $r_{i}$ from Eq. 9.

- Calculate $S^{2}$ from Eq. 7.

- Compute the visibility function $\mathrm{R}_{\mathrm{V}}$ from Eq. 11 , if

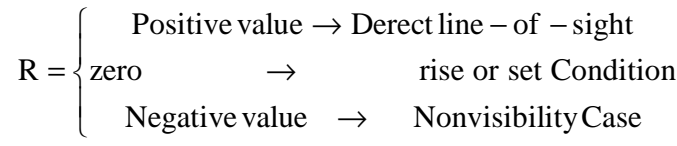

at the given time $\mathrm{t}$.

- The algorithm is completed

\section{RESULTS AND DISCUSSION}

A MATHEMATICA 8 programing language was designed and executed for calculating and plotting curves to test the behaviour of the sign of the visibility function $R_{V}$ from Eq. 11 applied for two satellites in the same orbit (have the same orbital elements except the mean anomaly) in two cases:

- If the difference in mean anomaly is $10^{\circ}$, logically the two satellites will be in a direct line-of-sight communication case all the time

- If the difference in mean anomaly is $180^{\circ}$, logically the two satellites will be in a nonvisibility case all the time

The real application on a geostationary satellites shows that: the previouse assumption was done in fact (see Fig. 4 and 5).

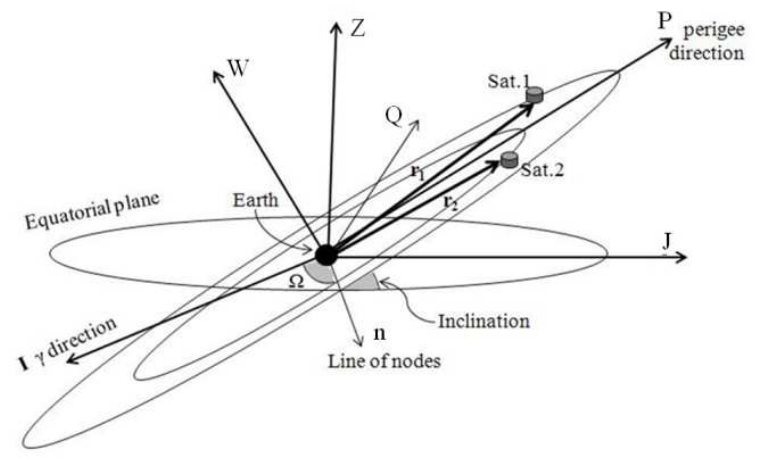

Fig. 3: The two satellites are in the same orbital plane

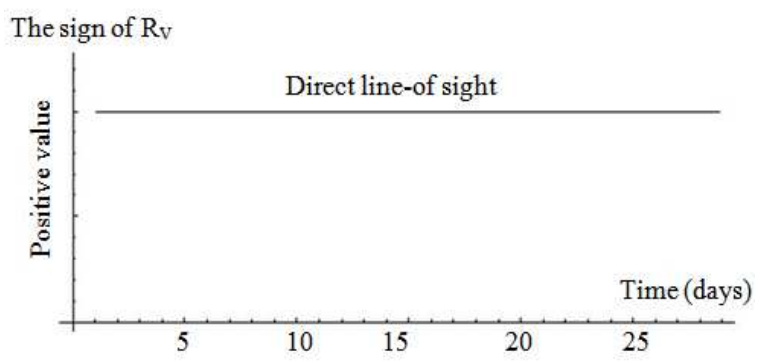

Fig. 4: The behaviour of the sign of $R_{V}$ with the time in case of the difference between two satellites in meananomaly is $10^{\circ}$ 
Am. J. Applied Sci., 9 (5): 620-623, 2012

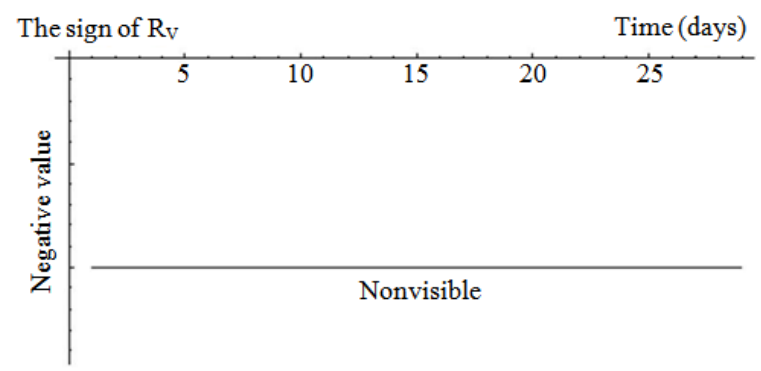

Fig. 5: The behaviour of the sign of $R_{V}$ with the time in case of the difference between two satellites in meananomaly is $180^{\circ}$

\section{CONCLUSION}

This study presented a method to rapidly determine the visibility of satellite-satellite visibility periods. This study calculated the problem by using an algorithm which used a simple treatment to construct the visibility function versus time. This algorithm was work for two satellites are in the same orbital plane.

Future work: Now, we are constructing a simple treatment to calculate the visibility function for two earth's satellites in different orbital planes.

\section{REFERENCES}

Alfano, S., D. Negron Jr. and J.L. Moore, 1992. Rapid determination of satellite visibility periods. J. Astronautical Sci., 40: 281-296.

Escobal, P.R., 1964. Rise and Set Time of a Satellite About an Oblate Planet. 1st Edn., University of California, Los Angeles-Engineering, pp: 46.

Lawton, J.A., 1986. A numerical method for rapidly determining satellite-satellite and satellite-ground station in-view periods. J. Guidance Navigation Control.

Roth, E.A., 1976. Rise-and-set time and maximum elevation of a satellite. ESA Sci. Tech. Rev., 2: 1124. 